\title{
LARGE-SCALE MAGNETIC FIELD IN ACCRETION DISKS AND RELATIVISTIC POYNTING-FLUX JETS
}

\author{
R. V. E. LOVELACE \\ Department of Astronomy, Cornell University, Ithaca, NY 14853-6801 \\ RVL1@cornell.edu \\ G. S. BISNOVATYI-KOGAN \\ Space Research Institute, Russian Academy of Sciences, Moscow, Russia \\ gkogan@mx.iki.rssi.ru
}

\begin{abstract}
In earlier works we pointed out that the disk's surface layers are non-turbulent and thus highly conducting (or non-diffusive) because of hydrodynamic and/or magnetorotational (MRI) instabilities are suppressed high in the disk where the magnetic and radiation pressures are larger than the plasma thermal pressure. We have derived equations for the vertical profiles of stationary accretion flows (with radial and azimuthal components), and the profiles of the large-scale, magnetic field taking into account the turbulent viscosity and diffusivity and the fact that the turbulence vanishes at the surface of the disk. Our recent analysis in Ref. 1 shows that the inward or outward advection of the largescale magnetic field depends on the ratio $\mathcal{R}$ of the accretion power going into magnetic disk winds to the viscous power dissipation and the plasma- $\beta$ which is the ratio of the midplane plasma pressure to the magnetic pressure.

Recent radio emission, polarization, and Faraday rotation maps of the radio jet of the galaxy 3C303 have been obtained in Ref. 2 and show that one component of this jet has a galactic-scale electric current of $\sim 3 \times 10^{18}$ A flowing along the jet axis. We show that this current can be used to calculate the electromagnetic energy flow in this magnetically dominated jet.
\end{abstract}

Keywords: accretion, accretion disks — galaxies: jets — magnetic fields — MHD

\section{Introduction}

In the first part of this paper we discuss the diffusion and advection of a large-scale field in an accretion disk with a turbulent viscosity comparable to the turbulent magnetic diffusivity (as suggested by MRI simulations) indicated that a weak largescale field would diffuse outward rapidly. Earlier in Ref. 3 we pointed out that the disk's surface layers are highly conducting (or non-diffusive) because the instabilities are suppressed in this region where the magnetic and radiative energy-densities are larger than the gas thermal energy-density. This was analyzed further in Ref. 4, and in Ref. 5 we developed an analytic model for the vertical $(z)$ profiles of the stationary accretion flows (with radial and azimuthal components), and the profiles of the large-scale, magnetic field taking into account the turbulent viscosity and 
diffusivity due to the MRI and the fact that the turbulence vanishes at the surface of the disk.

In the second part of this paper we discuss a relativistic Poynting-flux jet model for the observed current flow along the jet axis in the extra-galactic radio jet of the galaxy 3C 303 (Ref. 2).

Conclusions are given at the end of each section.

\section{Advection/Diffusion of Large-Scale B-Field in an Accretion Disk}

Following Ref. 5 we consider the non-ideal magnetohydrodynamics of a thin axisymmetric, viscous, resistive disk threaded by a large-scale dipole-symmetry magnetic field $\mathbf{B}$. We use a cylindrical $(r, \phi, z)$ inertial coordinate system in which the timeaveraged magnetic field is $\mathbf{B}=B_{r} \hat{\mathbf{r}}+B_{\phi} \hat{\boldsymbol{\phi}}+B_{z} \hat{\mathbf{z}}$, and the time-averaged flow velocity is $\mathbf{v}=v_{r} \hat{\mathbf{r}}+v_{\phi} \hat{\boldsymbol{\phi}}+v_{z} \hat{\mathbf{z}}$. The main equations are

$$
\begin{aligned}
\rho \frac{d \mathbf{v}}{d t} & =-\nabla p+\rho \mathbf{g}+\frac{1}{c} \mathbf{J} \times \mathbf{B}+\mathbf{F}^{\nu}, \\
\frac{\partial \mathbf{B}}{\partial t} & =\nabla \times(\mathbf{v} \times \mathbf{B})-\nabla \times(\eta \nabla \times \mathbf{B}) .
\end{aligned}
$$

These equations are supplemented by the continuity equation, $\nabla \cdot(\rho \mathbf{v})=0$, by $\nabla \times \mathbf{B}=4 \pi \mathbf{J} / c$, and by $\nabla \cdot \mathbf{B}=0$. Here, $\eta$ is the magnetic diffusivity, $\mathbf{F}^{\nu}=-\nabla \cdot T^{\nu}$ is the viscous force with $T_{j k}^{\nu}=-\rho \nu\left(\partial v_{j} / \partial x_{k}+\partial v_{k} / \partial x_{j}-(2 / 3) \delta_{j k} \nabla \cdot \mathbf{v}\right)$ (in Cartesian coordinates), and $\nu$ is the kinematic viscosity. For simplicity, in place of an energy equation we consider the adiabatic dependence $p \propto \rho^{\gamma}$, with $\gamma$ the adiabatic index.

We assume that both the viscosity and the diffusivity are due to magnetorotational (MRI) turbulence in the disk so that

$$
\nu=\mathcal{P} \eta=\alpha \frac{c_{s 0}^{2}}{\Omega_{K}} g(z)
$$

where $\mathcal{P}$ is the magnetic Prandtl number of the turbulence assumed a constant of order unity (Ref. 6), $\alpha \leq 1$ is the dimensionless Shakura-Sunyaev parameter, $c_{s 0}$ is the midplane isothermal sound speed, $\Omega_{K} \equiv\left(G M / r^{3}\right)^{1 / 2}$ is the Keplerian angular velocity of the disk, and $M$ is the mass of the central object. The function $g(z)$ accounts for the absence of turbulence in the surface layer of the disk (Refs. 3 and 4). In the body of the disk $g=1$, whereas at the surface of the disk, at say $z_{S}$, $g$ tends over a short distance to a very small value $\sim 10^{-8}$, effectively zero, which is the ratio of the Spitzer diffusivity of the disk's surface layer to the turbulent diffusivity of the body of the disk. At the disk's surface the density is much smaller than its midplane value.

We consider stationary solutions of equations (1) and (2) for a weak large-scale magnetic field. These can be greatly simplified for thin disks where the disk halfthickness, of the order of $h \equiv c_{s 0} / \Omega_{K}$, is much less than $r$. Thus we have the small parameter $\varepsilon=h / r=c_{s 0} / v_{K} \ll 1$. It is useful in the following to use the dimensionless height $\zeta \equiv z / h$. The midplane plasma beta is taken to be $\beta \equiv 4 \pi \rho_{0} c_{s 0}^{2} / B_{0}^{2}$, 
where $\beta=c_{s 0}^{2} / v_{A 0}^{2}, v_{A 0}=B_{0} /\left(4 \pi \rho_{0}\right)^{1 / 2}$ is the midplane Alfvén velocity. Note that the conventional definition of beta is $2 \beta$. The rough condition for the MRI instability and the associated turbulence in the disk is $\beta \gtrsim 1$ (Ref. 7) and this is assumed here.

The three magnetic field components are assumed to be of comparable magnitude on the disk's surface, but $B_{r}=0=B_{\phi}$ on the midplane. On the other hand the axial magnetic field changes by only a small almount going from the midplane to the surface, $\Delta B_{z} \sim \varepsilon B_{r} \ll B_{z}$ (from $\nabla \cdot \mathbf{B}=0$ ) so that $B_{z} \approx$ const inside the disk. As a consequence, the $\partial B_{j} / \partial r$ terms in the magnetic force in equation (1) can all be dropped in favor of the $\partial B_{j} / \partial z$ terms (with $j=r, \phi$ ). It is important to keep in mind that $B_{j}$ is the large scale field; the approximation does not apply to the small-scale field which gives the viscosity and diffusivity. The three velocity components are assumed to satisfy $v_{z}^{2} \ll c_{s 0}^{2}$ and $v_{r}^{2} \ll v_{\phi}^{2}$. Consequently, $v_{\phi}(r, z)$ is close in value to the Keplerian value $v_{K}(r) \equiv(G M / r)^{1 / 2}$, except in the outer disk layers where the radial magnetic force may be comparable with the centrifugal and gravitational forces. We normalize the field components by $B_{0}=B_{z}(r, z=0)$, with $b_{r}=B_{r} / B_{0}, b_{\phi}=B_{\phi} / B_{0}$, and $b_{z}=B_{z} / B_{0} \approx 1$. Also, we define $u_{\phi} \equiv v_{\phi}(r, z) / v_{K}(r)$ and the accretion speed $u_{r} \equiv-v_{r} /\left(\alpha c_{s 0}\right)$. For the assumed dipole field symmetry, $b_{r}$ and $b_{\phi}$ are odd functions of $\zeta$ whereas $u_{r}$ and $u_{\phi}$ are even functions. We assume $p \propto \rho^{\gamma}$ so that the vertical hydrostatic equilibrium gives $\tilde{\rho}=\rho / \rho_{0}$ as a function of $\zeta$ which goes to zero at $\zeta_{m}$. However, before this distance is reached the MRI turbulence is suppressed, and $g(\zeta)$ in equation (3) is effectively zero.

Integration over the vertical extent of the disk gives the average accretion speed

$$
\bar{u}_{r}=u_{0}-\frac{2 b_{\phi S+}}{\alpha \beta \tilde{\Sigma}}
$$

(Ref. 5) which is the sum of a viscous contribution, $u_{0} \equiv 3 \varepsilon k_{\nu}$ (with $k_{\nu}$ a numerical constant of order unity), and a magnetic contribution $\left(\propto b_{\phi S+}\right)$ due to the loss of angular momentum from the surface of the disk where necessarily $b_{\phi S+} \leq 0$ (Ref. $8)$.

The different components of equations (1) and (2) can be combined to give the following equation for the radial accretion speed,

$$
\begin{array}{r}
\alpha^{4} \beta^{2} \frac{\partial^{2}}{\partial \zeta^{2}}\left(g \frac{\partial}{\partial \zeta}\left(\tilde{\rho} g \frac{\partial}{\partial \zeta}\left(\frac{1}{\tilde{\rho}} \frac{\partial}{\partial \zeta}\left(\tilde{\rho} g \frac{\partial u_{r}}{\partial \zeta}\right)\right)\right)\right) \\
-\alpha^{2} \beta \mathcal{P} \frac{\partial^{2}}{\partial \zeta^{2}}\left(g \frac{\partial}{\partial \zeta}\left(\tilde{\rho} g \frac{\partial}{\partial \zeta}\left(\frac{u_{r}}{\tilde{\rho} g}\right)\right)\right) \\
-\alpha^{2} \beta \mathcal{P} \frac{\partial^{2}}{\partial \zeta^{2}}\left(\frac{1}{\tilde{\rho}} \frac{\partial}{\partial \zeta}\left(\tilde{\rho} g \frac{\partial u_{r}}{\partial \zeta}\right)\right) \\
+\alpha^{2} \beta^{2} \frac{\partial^{2}}{\partial \zeta^{2}}\left(\tilde{\rho} g\left(u_{r}-g u_{0}\right)\right)+\mathcal{P}^{2} \frac{\partial^{2}}{\partial \zeta^{2}}\left(\frac{u_{r}}{\tilde{\rho} g}\right) \\
+3 \beta \mathcal{P}^{2} \frac{u_{r}}{g}=0,
\end{array}
$$




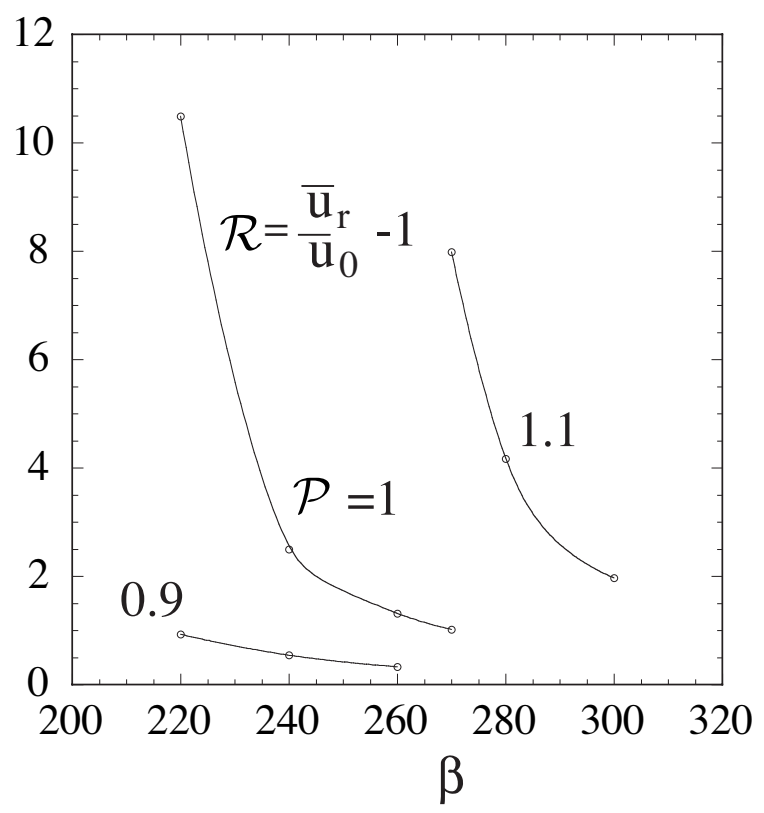

Fig. 1. Summary of internal disk solutions which satisfy the boundary conditions from Ref. 1. $\bar{u}_{r}$ is the average accretion speed and $u_{0}$ is the accretion speed in the absence of an ordered magnetic field. The curves are smooth lines connecting the marked points. In this plot $\varepsilon=h / r=0.05$, and viscosity parameter $\alpha=0.1$.

(Ref. 5). The equation can be integrated from $\zeta=0$ out to the surface of the disk $\zeta_{S}$ where boundary conditions apply.

In general there is a continuum of values of $b_{\phi S} \leq 0$ for the considered solutions inside the disk. The value of $b_{\phi S}$ can be determined by matching the calculated fields $b_{r S}$ and $b_{\phi S}$ onto an external field and flow. Earlier, Ref. 5 showed that there is no jump in $b_{r}$ across the conducting surface layer. This implies that $\partial u_{r} /\left.\partial \zeta\right|_{\zeta_{S}}=0$. which represents a first boundary condition. A second boundary condition, $\left.u_{r}\right|_{\zeta_{S}}=0$ follows from equation 12 of Ref. 5 evaluated just outside of the conducting layer. A third boundary condition, $\partial b_{\phi} /\left.\partial \zeta\right|_{\zeta_{S-}}=0$, is derived in Ref. 5 .

In addition to the three boundary conditions at the surface of the disk, there is the unknown ratio $\mathcal{R}$ of the accretion power going into magnetic disk winds to the viscous power dissipation. The solution of eqn. 5 for $u_{r}(\zeta)$ has three unknown coefficients which are dependent on $\alpha, \mathcal{P}$, and $\beta$. If $\alpha$ and $\mathcal{P}$ are fixed, then there is a definite relation between $\mathcal{R}$ and $\beta$. Figure 1 shows this relation determined numerically.

The value of $b_{\phi S+} \leq 0$ or $\bar{u}_{r} / u_{0}$ is not fixed by the solution for the field and flow inside the disk. Its value can be determined by matching the calculated surface fields $b_{r S}$ and $b_{\phi S+}$ onto an external magnetic wind or jet solution. Stability of the 
wind or jet solution to current driven kinking is predicted to limit the ratio of the toroidal to axial magnetic field components at the disk's surface $\left|b_{\phi S+}\right|$ to values $\lesssim \mathcal{O}(2 \pi)$ (Ref. 5). Recall that $\mathcal{R}=\bar{u}_{r} / u_{0}-1=2\left|b_{\phi S+}\right| /\left(\alpha \beta \sqrt{2} u_{0}\right)$ is the ratio of the accretion power going into the disk wind to the viscous dissipation in the disk. For the mentioned upper limit on $\left|b_{\phi S+}\right|, \mathcal{R} \lesssim \mathcal{O}\left[2 \sqrt{2} \pi /\left(\alpha \beta u_{0}\right)\right] \approx 2.5$. This implies a critical value of $\beta$ for a stationary disk solution for $\mathcal{P}=1, \beta_{c} \approx 1.2(\alpha \varepsilon)^{-1}$. For $\alpha=0.1$ and $\varepsilon=0.05, \beta_{c} \approx 240$.

For $\beta \lessgtr \beta_{c}$, the disk and large-scale magnetic field is not be in a stationary state. Equation (4) for the average accretion speed still applies and can be written as $\bar{u}_{r}=u_{0}+u_{B}$ where $u_{B}$ is the magnetic contribution to the accretion due to the outflow of angular momentum from the disk's surfaces. In general, $u_{B}$ is an increasing function of the magnetic field strength if $\mathcal{R} \sim 1$ (Ref. 8). For this reason for $\beta<\beta_{c}$ the ordered poloidal magnetic field threading the disk will be advected inward in the disk while for $\beta>\beta_{c}$ the field will advect outward. Thus, $\beta_{c}$ acts as a threshold value for the buildup of a large scale field in the inner regions of an accretion disk.

Another possibility is that the disk winds are negligible so that $\mathcal{R} \ll 1$. Figure 1 suggests that in this limit we have a stable stationary solutions with $\beta$ values much larger than the mentioned critical value.

The 3D MHD simulations of Ref. 9 shows the inward advection of a significant fraction of the initial unipolar vertical magnetic flux threading a MRI unstable plasma torus around a black hole. The initial average $\beta$ in the torus is 100 which is significantly less than the critical value estimated here. A broader range of simulations for much larger initial values of $\beta$ would be needed to determine if the initial flux diffuses outward.

\section{Relativistic Poynting-Flux Jets}

Radio emission, polarization, and Faraday rotation maps of the radio jet of the galaxy 3C303 have been obtained in Ref. 2 and show that one component of this jet has a galactic-scale electric current $\left(\sim 3 \times 10^{18} \mathrm{~A}\right)$ flowing along the jet axis. This current can be interpreted in terms of a relativistic Poynting-flux jet. The discussion here is an extension of our earlier work in Refs. 10-13.

In cylindrical $(r, \phi, z)$ coordinates with axisymmetry assumed the magnetic field has the form $\mathbf{B}=\mathbf{B}_{p}+B_{\phi} \hat{\boldsymbol{\phi}}$, with $\mathbf{B}_{p}=B_{r} \hat{\mathbf{r}}+B_{z} \hat{\mathbf{z}}$, and $B_{r}=-(1 / r)(\partial \Psi / \partial z)$, and $B_{z}=(1 / r)(\partial \Psi / \partial r)$. Here, $\Psi(r, z) \equiv r A_{\phi}(r, z)$ is the flux function. A representative form of this function is $\Psi(r, 0)=(1 / 2) r^{2} B_{0} /\left[1+2\left(r / r_{0}\right)^{3}\right]$, where $B_{0}$ is the axial magnetic field strength in the center of the disk, and $r_{0}$ is the radius of the $O$-point of the magnetic field in the plane of the disk as indicated in Figure 2. This field could arise for $t \geq 0$ from dynamo processes in the disk triggered by a star-disk collision as discussed in Ref. 14. The field may be sufficiently strong $(\beta<1)$ that it suppresses the MRI instability so that the disk is non-turbulent and without viscosity. The disk will however continue to slowly accrete owing to the angular momentum outflow in 

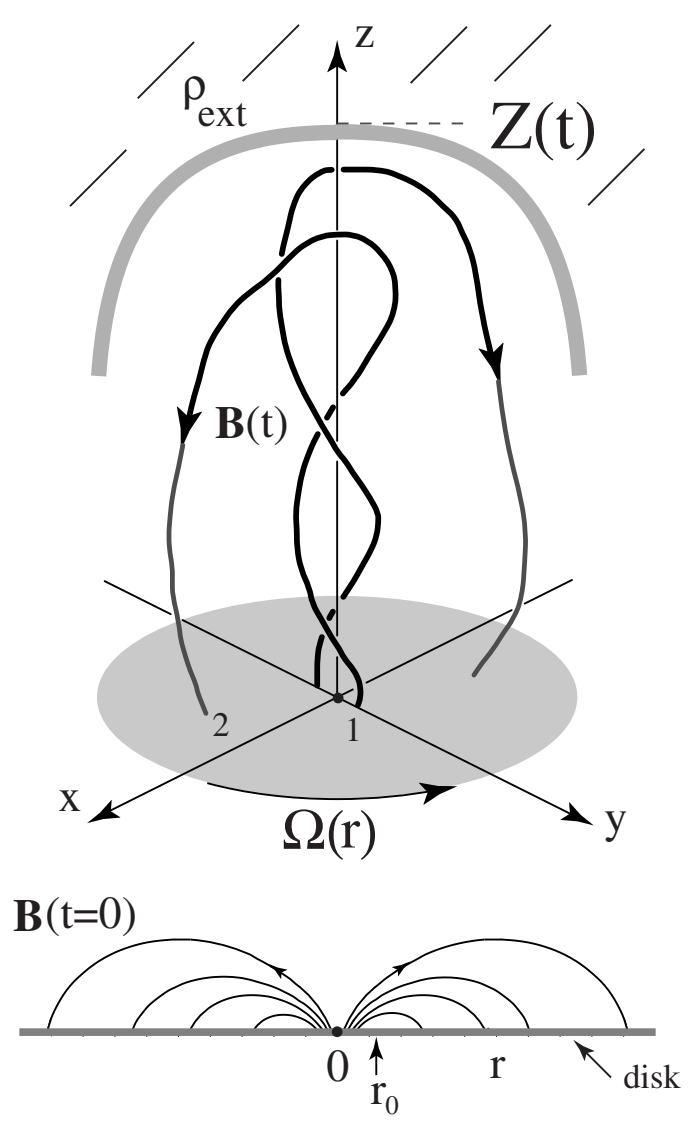

Fig. 2. Sketch of the magnetic field configuration of a Poynting jet adapted from Ref. 10. The bottom part of the figure shows the initial dipole-like magnetic field threading the disk which rotates at the angular rate $\Omega(r)$. The top part of the figure shows the jet at some time later when the head of the jet is at a distance $Z(t)$. At the head of the jet there is force balance between electromagnetic stress of the jet and the ram pressure of the ambient medium of density $\rho_{\text {ext }}$.

the Poynting jet which gives a radial accretion speed much less than the Keplerian velocity of the disk. This $\Psi$ is taken to apply for $r \geq 0$ even though it is not valid near the horizon of the black hole. The contribution from this region is negligble for the considered conditions where $\left(r_{g} / r_{0}\right)^{2} \ll 1$, where $r_{g} \equiv G M / c^{2}$. For a corotating disk around a Kerr black hole the disk's angular velocity viewed from a large distance is $\Omega=\left[c^{3} /(G M)\right] /\left[a_{*}+\left(r / r_{g}\right)^{3 / 2}\right]$, for $r>r_{m s}$ where $r_{\mathrm{ms}}$ is the innermost stable circular orbit and $a_{*}$ is the spin parameter of the black hole with $0 \leq a_{*}<1$.

The space above (and below) the disk is assumed to be filled with a low-density 'coronal' plasma which is "force-free" in the sense that its kinetic energy-density is much smaller than the magnetic energy density. For this plasma we have a continuity equation, Ampère's law, Coulomb's law, Faraday's law, perfect conductivity, $\mathbf{E}+$ 
$\mathbf{u} \times \mathbf{B} / c=0$, with $\mathbf{u}$ the plasma flow velocity, and the "force-free" condition in the Euler equation, $\rho_{e} \mathbf{E}+\mathbf{J} \times \mathbf{B} / c=0$. Owing to the assumed axisymmetry, $E_{\phi}=0$, so that the poloidal velocity $\mathbf{u}_{p}=\kappa \mathbf{B}_{p}$. Mass conservation then gives $\mathbf{B} \cdot \nabla(\rho \kappa)=0$, which implies that $\rho \kappa=F(\Psi) / 4 \pi$, where $F$ is an arbitrary function of $\Psi$. In a similar way one finds that $u_{\phi}-\kappa B_{\phi}=r G(\Psi)$, so that $\mathbf{E}=-G(\Psi) \nabla \Psi$, and $r B_{\phi}=H(\Psi)$, so that there are two additional functions, $G$ and $H$. The function $G$ is determined along all of the field lines which go through the disk. This follows from the perfect conductivity condition at the surface of the disk, $z=0, E_{r}+(\mathbf{u} \times \mathbf{B})_{r} / c=0$. This gives $E_{r}=-\left(u_{\phi} B_{z}-u_{z} B_{\phi}\right) / c=-u_{\phi} B_{z} / c$, where $u_{z}$ is zero at the disk and $u_{\phi}$ is the disk velocity. Therefore, $E_{r}(r, 0)=-\Omega[d \Psi(r, 0) / d r] / c$, so that $G(\Psi)=\Omega(r) / c$ which gives $\Omega=\Omega(\Psi)$.

The component of the Euler equation in the direction of $\nabla \Psi$ gives the force-free Grad-Shafranov (GS) equation,

$$
\left[1-\left(\frac{r \Omega}{c}\right)^{2}\right] \Delta^{\star} \Psi-\frac{\nabla \Psi}{2 r^{2}} \cdot \nabla\left(\frac{r^{4} \Omega^{2}}{c^{2}}\right)+H(\Psi) \frac{d H(\Psi)}{d \Psi}=0,
$$

with $\Delta^{\star} \equiv \partial^{2} / \partial r^{2}-(1 / r)(\partial / \partial r)+\partial^{2} / \partial z^{2}$ (Ref. 15).

We consider an initial value problem where the disk at $t=0$ is threaded by a dipole-like poloidal magnetic field. The form of $H(\Psi)$ in equation (6) is then determined by the differential rotation of the disk: The azimuthal twist of a given field line going from an inner footpoint at $r_{1}$ to an outer footpoint at $r_{2}$ is fixed by the differential rotation of the disk.

Ampère's law gives $\oint d \mathbf{l} \cdot \mathbf{B}=(4 \pi / c) \int d \mathbf{S} \cdot \mathbf{J}$, so that $r B_{\phi}(r, z)=H(\Psi)$ is $(2 / c)$ times the current flowing through a circular area of radius $r$ (with normal $\hat{\mathbf{z}})$ labeled by $\Psi(r, z)=$ const. Equivalently, $-H[\Psi(r, 0)]$ is $(2 / c)$ times the current flowing into the area of the disk $\leq r$. Our previous work in Ref. 11 shows that $-H(\Psi)$ has a maximum so that the total current flowing into the disk for $r \leq r_{m}$ is $I_{\text {tot }}=(c / 2)(-H)_{\max }$, where $r_{m}$ is such that $-H\left[\Psi\left(r_{m}, 0\right)\right]=(-H)_{\max }$ where $r_{m}$ is less than the radius of the $O$-point, $r_{0}$. The same total current $I_{\text {tot }}$ flows out of the region of the disk $r=r_{m}$ to $r_{0}$.

For a given field line we have $r d \phi / B_{\phi}=d s_{p} / B_{p}$, where $d s_{p} \equiv \sqrt{d r^{2}+d z^{2}}$ is the poloidal arc length along the field line, and $B_{p} \equiv \sqrt{B_{r}^{2}+B_{z}^{2}}$. The total twist of a field line loop is

$$
\Delta \phi(\Psi)=-\int_{1}^{2} d s_{p} \frac{B_{\phi}}{r B_{p}}=-H(\Psi) \int_{1}^{2} \frac{d s_{p}}{r^{2} B_{p}},
$$

with the sign included to give $\Delta \phi>0$. The integration goes from the disk at a radius $r_{1}<r_{0}$ out into the corona and back to the disk at a radius $r_{2}>r_{0}$. For a prograde disk the field line twist after a time $t$ is

$$
\Delta \phi(\Psi)=\Omega\left(r_{0}\right) t\left[\frac{\Omega\left(r_{1}\right)}{\Omega\left(r_{0}\right)}-\frac{\Omega\left(r_{2}\right)}{\Omega\left(r_{0}\right)}\right] .
$$

At sufficiently small $r_{1}$ one reaches the inner radius of the disk $r_{i}$ where $\Omega$ is assumed to saturate at the value $\Omega_{i}=\Omega\left(r_{i}\right)$. 
Our previous study of non-relativstic Poynting jets by analytic theory in Ref. 11 and axisymmetric MHD simulations in Ref. 13 showed that as the twist, as measured by $\Omega\left(r_{0}\right) t$, increases a new, high twist field configuration appears with a different topology. A "plasmoid" consisting of toroidal flux detaches from the disk and propagates outward. The plasmoid is bounded by a poloidal field line which has an $X$-point above the $O$-point on the disk. The occurrence of the $X$-point requires that there be at least a small amount of dissipation in the evolution from the poloidal dipole field and the Poynting jet configuration. The high-twist configuration consists of a region near the axis which is magnetically collimated by the toroidal $B_{\phi}$ field and a region far from the axis which is anti-collimated in the sense that it is pushed away from the axis. The field lines returning to the disk at $r>r_{0}$ are anti-collimated by the pressure of the toroidal magnetic field. The poloidal field fills only a small part of the coronal space.

Relativistic Poynting jets are expected to have a magnetic field configuration similar to that observed in the non-relativistic limit. That is, most of the twist $\Delta \phi$ of a field line of a relativistic Poynting jet occurs along the jet from $z=0$ to $Z(t)$ as sketched in Figure 2, where $Z(t)$ is the axial location of the "head" of the jet. Along most of the distance $z=0-Z$ the radius of the jet is a constant and $\Psi=\Psi(r)$ for $Z \gg r_{0}$. Note that the function $\Psi(r)$ is different from $\Psi(r, 0)$ which is the flux function profile on the disk surface. Hence,

$$
r^{2} \frac{d \phi}{d z}=\frac{r B_{\phi}(r, z)}{B_{z}(r, z)} .
$$

For simplicity we consider $U_{z}=d Z / d t=$ const. In this case, equations (7) and (8) give $H(\Psi)=\left[r^{2} \Omega(\Psi) / U_{z}\right] B_{z}$. Thus, equation (6) can be written as

$$
\frac{d^{2} \Psi}{d r^{2}}+\frac{\lambda-1}{\lambda+1} \frac{1}{r} \frac{d \Psi}{d r}+\frac{\lambda}{\lambda+1}\left(\frac{d \Psi}{d r}\right)^{2} \frac{1}{\Omega} \frac{d \Omega}{d \Psi}=0
$$

where $\lambda \equiv(r \Omega / c)^{2}\left[\left(c / U_{z}\right)^{2}-1\right]=(r \Omega / c)^{2} /\left(\Gamma^{2}-1\right)$, and where $\Gamma \equiv\left(1-U_{z}^{2} / c^{2}\right)^{-1 / 2}$. For the radio jets of active galaxies $\Gamma^{2} \gg 1$ for distances $Z \lesssim 1 \mathrm{kpc}$ (Ref. 10).

Solution of equation (10) is facilitated by introducing dimensionless variables. We measure the radial distance in units of the distance $r_{0}$ to the $O$-point of the magnetic field threading the disk indicated in Fig. 2. We measure the flux function $\Psi$ in units of $\Psi_{0} \equiv r_{0}^{2} B_{0} / 2$. The fields are measured in units of $B_{0}$ which is the magnetic field strength at the center of the disk. Thus, $\bar{B}_{z}=(2 \bar{r})^{-1} d \bar{\Psi} / d \bar{r}$. The disk rotation rate $\Omega$ is measured in units of $c^{3} /(G M)$. In terms dimensionless variables $\bar{r}, \bar{\Psi}$, and $\bar{\Omega}$, equation (10) is the same with overbars on these three variables. Note also that $\lambda=\bar{r}^{2} \mathcal{R}^{2} \bar{\Omega}^{2} /\left(\Gamma^{2}-1\right)$, where $\mathcal{R} \equiv r_{0} / r_{g} \geq 1$.

We find solutions of equation (10) using the approximation $\bar{\Psi}(r, 0)=\bar{r}^{2}$ which gives $\bar{\Omega}=1 /\left(a_{*}+\mathcal{R}^{3 / 2} \bar{\Psi}^{3 / 4}\right)$. These solutions are close to those obtained using the full dependence $\Omega(\Psi)$. In this approximation there is a unique self-similar solution 
to equation (10) for $\bar{\Psi} \gg 1 / \mathcal{R}^{2}$ where $\bar{\Omega} \approx \mathcal{R}^{-3 / 2} \bar{\Psi}^{-3 / 4}$. This solution is

$$
\bar{\Psi}=\frac{\bar{r}^{4 / 3}}{\left[2 \mathcal{R}\left(\Gamma^{2}-1\right)\right]^{2 / 3}}, \quad \text { and } \quad \lambda=2 .
$$

This dependence holds for

$$
\bar{r}_{1}=\frac{\left[2\left(\Gamma^{2}-1\right)\right]^{1 / 2}}{\mathcal{R}}<\bar{r}<\bar{r}_{2}=\frac{\left[2 \mathcal{R}\left(\Gamma^{2}-1\right)\right]^{1 / 2}}{3^{3 / 4}} .
$$

At the inner radius $\bar{r}_{1}, \bar{\Psi}=1 / \mathcal{R}^{2}$, which corresponds to the streamline which passes through the disk at a distance $r=r_{g}$. For $\bar{r}<\bar{r}_{1}$, we assume $\bar{\Psi} \propto \bar{r}^{2}$, which corresponds to $B_{z}=$ const. At the outer radius $\bar{r}_{2}, \bar{\Psi}=(\bar{\Psi})_{\max }=1 / 3$ which corresponds to the streamline which goes through the disk near the $O$-point at $r=r_{0}$. Note that there is an appreciable range of radii if $\mathcal{R}^{3 / 2} \gg 1$.

For $\bar{r}_{1}<r<\bar{r}_{2}$, the field components of the Poynting jet,

$$
\bar{E}_{r}=-\sqrt{2}\left(\Gamma^{2}-1\right)^{1 / 2} \bar{B}_{z}, \quad \bar{B}_{\phi}=-\sqrt{2} \Gamma \bar{B}_{z}, \quad \bar{B}_{z}=\frac{2}{3} \frac{\bar{r}^{-2 / 3}}{\left[2 \mathcal{R}\left(\Gamma^{2}-1\right)\right]^{2 / 3}} .
$$

This electromagnetic field statisfies the radial force balance equation, $d B_{z}^{2} / d r+$ $\left(1 / r^{2}\right) d\left[r^{2}\left(B_{\phi}^{2}-E_{r}^{2}\right)\right] / d r=0$ as it should. At the outer radius of the jet at $r_{2}$, there is a boundary layer where the axial field changes from $B_{z}\left(r_{2}\right)$ to zero while (minus) the toroidal field increases by a corresponding amount so as to satisfy the radial force balance. This gives $-r_{2} B_{\phi}\left(r_{2}\right)=(-H)_{\max }=2 I_{\max } / c=(2 / \sqrt{3}) \Psi\left(r_{2}\right) / r_{2}$. Thus for $r>r_{2}, E_{r}=0, B_{\phi}=\sqrt{3} B_{z}\left(r_{2}\right)\left(r_{2} / r\right)$, and $B_{z}=0$. These fields apply out to a radius $r_{3}$ where $B_{\phi}^{2}\left(r_{3}\right) / 8 \pi=p_{\mathrm{ex}}$, where $p_{\mathrm{ex}}=n_{\mathrm{ex}} k_{\mathrm{B}} T_{\mathrm{ex}}$ is the pressure in the external medium.

The axial current $I$ carried by the Poynting jet follows from Ampère's law, $B_{\phi}\left(r_{2}\right)=2 I /\left(c r_{2}\right)$, or in convenient units, $B_{\phi}[\mathrm{G}]=I[\mathrm{~A}] /(5 r[\mathrm{~cm}])$. Using equations (11)-(13), the energy flux carried by the Poynting jet can be expressed as

$$
\dot{E}_{J}=(c / 2) \int_{0}^{r_{2}} r d r E_{r} B_{\phi}=I^{2} Z, \quad \text { where } \quad \mathcal{Z}=\frac{3}{c}\left(1-\Gamma^{-2}\right)^{1 / 2},
$$

for $r_{1} \ll r_{2}$. Here, $\mathcal{Z}$ is the impedance of the Poynting jet in cgs units. The conversion to SGI units is $c^{-1} \rightarrow(4 \pi)^{-1}\left(\mu_{0} / \epsilon_{0}\right)^{1 / 2}=30 \Omega$. Earlier, the impedance of a relativistic Poynting jet was estimated in Ref. 16 to be $\sim c^{-1}$. For the observed axial current in the E3 jet component of $3 \mathrm{C} 303,3 \times 10^{18} \mathrm{~A}$ (Ref. 2), the electromagnetic energy flux is $\dot{E}_{J} \approx 8 \times 10^{45}\left(u_{z} / c\right) \mathrm{erg} \mathrm{s}^{-1}$. This energy flux is much larger than the photon luminosity of the jet of $3.7 \times 10^{41} \mathrm{erg} \mathrm{s}^{-1}$ integrated over $10^{8}$ to $10^{17}$ $\mathrm{Hz}$ (Ref. 2) assuming $u_{z} / c$ is not much smaller than unity.

\section{Acknowledgments}

R.V.E.L thanks M.M. Romanova for valuable discussions. He was supported in part by NASA grants NNX10AF63G and NNX11AF33G and by NSF grant AST1008636. The work of G.S.B.-K. was partially supported by RFBR grants 0802-00491 and 08-02-90106, RAN Program "Formation and evolution of stars and galaxies." 


\section{References}

1. G. S. Bisonvatyi-Kogan and R. V. E. Lovelace, submitted to Astrophys. J. (2011).

2. P. P. Kronberg et al., Astrophys. J. Lett., in press, (2011).

3. G. S. Bisnovatyi-Kogan and R. V. E. Lovelace, Astrophys. J. Lett. 667, L167 (2007).

4. D. M. Rothstein and R. V. E. Lovelace, Astrophys. J. 677, 1221, (2008).

5. R. V. E. Lovelace, D. M. Rothstein and G. S. Bisnovatyi-Kogan, Astrophys. J. 701, $885,(2009)$.

6. X. Guan and C. F. Gammie, Astrophys. J. 697, 1901 (2009).

7. S. A. Balbus and J. F. Hawley, Rev. Mod. Phys. 70, 1 (1998).

8. R. V. E. Lovelace, M. M. Romanova and W. I. Newmman, Astrophys. J. 437, 136 (1994).

9. K. Beckwith, J. F. Hawley and J. H. Krolik, Astrophys. J. 707, 428 (2009).

10. R. V. E. Lovelace and M. M. Romanova, Astrophys. J. Lett., 596, L159 (2003).

11. R. V. E. Lovelace et al., Astrophys. J. 572, 445 (2002).

12. H. Li et al., Astrophys. J. 561, 915 (2001).

13. G. V. Ustyugova et al., Astrophys. J. Lett., 541, L21 (2000).

14. V. I. Pariev et al., Astrophys. J. 658, 129 (2007).

15. E. T. Scharlemann and R. V. Wagoner, Astrophys. J. 182, 951, (1973).

16. R. V. E. Lovelace, Nature 262, 649 (1976). 